NASA Technical Memorandum 103175

AIAA-90-2222

\title{
Analysis and Design of Optimized Truncated Scarfed Nozzles Subject to External Flow Effects
}

Rickey J. Shyne

Lewis Research Center

Cleveland, Ohio

and

Theo G. Keith, Jr.

University of Toledo

Toledo, Ohio

Prepared for the

26th Joint Propulsion Conference

cosponsored by the AIAA, SAE, ASME, and ASEE

Orlando, Florida, July 16-18, 1990

\section{NASก}

(NASA-TM-10?175) AVALYSIS ANO OESISA LF

$\sqrt{90-25190}$

JPTIMILFU TPUNCATFU JCARFEU NOZZLES JUSJECT

$T$ TXTIRNAL FUN TFECTS (NASA) I?

$$
\text { CSCl DIA g3/0, unclas }
$$





\title{
ANALYSIS AND DESIGN OF OPTIMIZED TRIJNCATED SCARFED NOZZLES SUBJECT TO EXTERNAL FLOW EFFECTS
}

\author{
Rickey J. Shyne \\ INASA Lewis Research Center, Cloveland, Ohio \\ Theo G. Keith, Jr.* \\ Department of Mochanical Engineering \\ University of Toledo, Taledo, Ohio
}

\begin{abstract}
Reo's method for computing optimum thrust nozzles is modified to study the offects of axternal flow on the performance of a class of exhaust nozzles. Members of this class are termed scarfed nozzles. These are two-dimensional, nonsymmetric nozzles with a flat lower wall. The lower wall (the cowl) is truncated in order to save weight. Results from a parametric investigation are presented to show the effects of the external flowfield on performance.
\end{abstract}

\section{INTRODUCTION}

Over the past few years, interest has grown in hypersonic and supersonic cruise vehicle design. Research studies are currently being pertormed to evaluate the feasibility of advanized aircraft such as the high-speed civil transport. From these studies it has become clear that the exhaust niszzle of the vehicles will have to be optimized for thrust while restricting length for overall weight reduction.

Optimization of exhaust nozzle:s has been an ongoing research topic for many years. Early attempls consisted mainly of trial and error procedures for assumed nozzle shapes, lip angles, exit velocities, etc. Considerable judgement was needed to arrive at an "optimized" nozzle. A more systematic approach was developed by Rao (1). Variational calculus and the method of characteristics were used in order to compute the contour. Recently, Rao's method was modilied and assembled into a computer pregram by Nickerson (2). Rao's method was originally formulated for axisymmetric nozzles with circular throats as illustrated in Fig. 1. However, because of renewed interest in ramjet engine applications, the method was rodified to compute iwodimensional, flat, rectangular throat nozzles. Such a nozzle is shown in Fig. 2. The method was also applied to designs where the nozzle lower wall (the cowl) is terminated at the point where the last characteristic that emanates from the upper nozzle wall (the ramp) intersects the cowl. This defines a two-dimensional, nonsymmetric nozzle called a scarfed nozzle. The flat throat modification incorporated into the analysis assumes an initial expansion of uniform flow followed by a Prandil-Meyer expansion at the beginning of the tuming section.

Significantly reduced vehicle weights can be oblained with two-dimensional, nonsymmetric nozzles and further weight reduction can be achieved by truncating the nozzle cowl upstream of the last nozzle ramp characteristic. However, because of this truncation the design will be affected by the extemal flow as illustrated in Fig. 3. If the nozzle flow is underexpanded, an oblique shock wave and slip line will form at the cowl truncation point due to the interaction of the external and internal flows. The incorporation of the external flow effects into the optimization of the exhaust nozzle contour defines the problem which is presented in this paper.

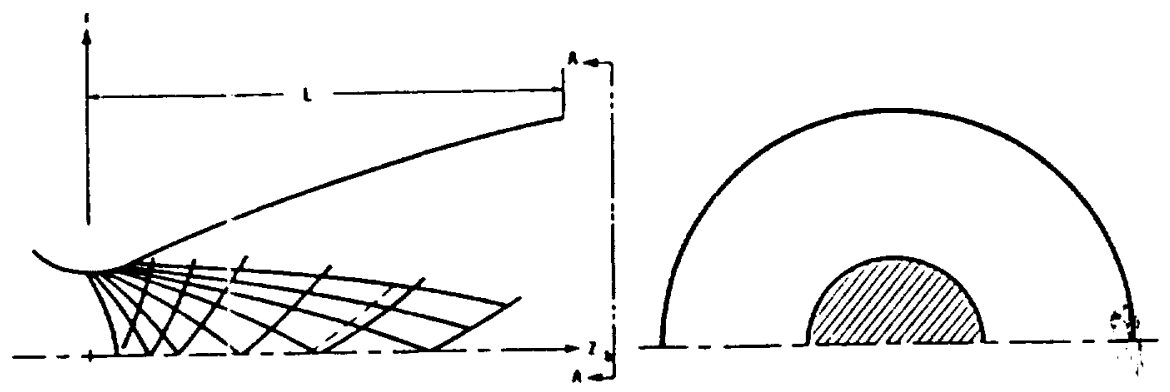

Fig. 1 - SCHEMATIC OF AN AXISWMMETRIC RAO NOZZLE

* MASA Resident Research Associate at Lewis Research Center. 


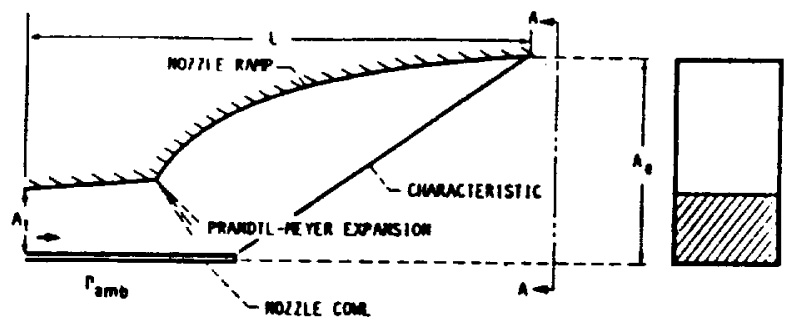

Flo. 2 - SCHEMATIC OF A TWODIMENSIONAL RAONOZAE WITH FLAT-THAOAT MOOIFICATION

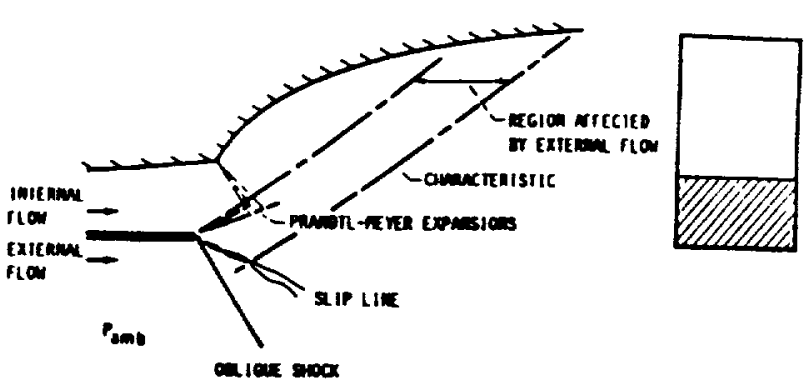

FD. 3 - SCHEMATIC OF A TWO-OMENSIONUL TRUNCATED RAO SCARFED NOZZL

The thrust on the nozzle is given by

$$
T=\int_{c}^{r} \theta\left[(P-P)+\rho r^{2} \frac{\sin (\varphi-\theta) \cos \theta}{\sin \varphi}\right] 2 \pi r d r
$$

method defines a nozzle contour for an ideal gas with constant specific heats in an isentropic flow. For this method a control surface is defined at the exit of the nozzle. The thrust is maximized such that the flow and the nozzle length are fixed constraints. The solution of the external problem and the flow within the nozzle are then found by using the method of characteristics.

Figure 4 is a schematic diagram of the upper portion of an axisymmetric nozzle contour with the characteristic net and control surface used in this analysis superimposed. Line CE describes the control surface having an angle of inclination to the axis. Figure 5 is a differential element of the control surface showing the flow across it. The mass flow rate through this element is given by

$$
d \dot{m}=\rho v \sin (\phi-\theta) d A
$$

where $d A=2 \pi r d s$ and $d s=d r / s i n \phi$. To obtain the mass flow crossing the control surface the differential mass Now rate is integrated along the line CE to produce

$$
\dot{m}=\int_{c}^{r} \rho v \frac{\sin (\phi-\theta)}{\sin \phi} 2 \pi r d r
$$

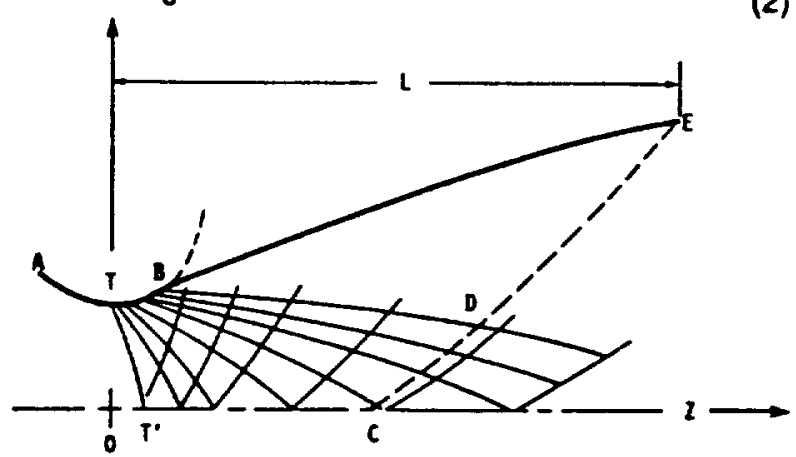

Fig. 4 - SCHEMATIC OF A RAO NOZZLE WTH CHARACTERISTIC NET AND CONTROL SURFACE

$$
\int_{t_{c}}^{r} \cot \phi d t=\text { constant }
$$

For a fixed throat contour, the length of the nozzle to point $C, Z_{C}$ is also fixed by the application of the entrance flow character and the solution of the flow equations. Hence the length constraint becomes

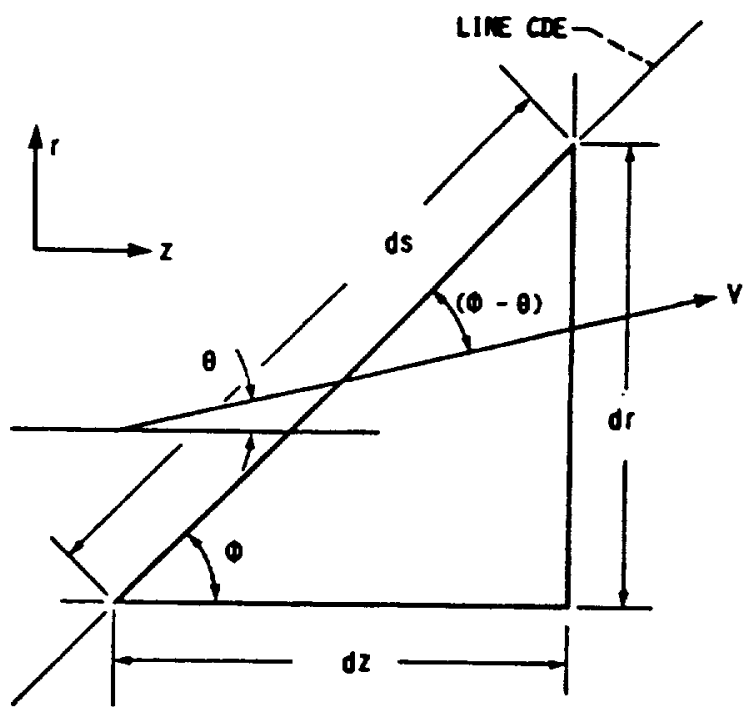
SURFACE
Fig. 5 - DIAGRAM OF A DIFFERENTUL ELEMENT OF CONTROL 
Utilizing the Lagrangian multiplier technique, see $\mathbf{\theta . g}$.. Hildebrand (3), the problem can be reduced to maximizing the following integral

$$
l=\int_{r_{e}}^{r_{0}}\left(f_{1}+\lambda_{1} f_{2}+\lambda_{3} f_{3}\right) d r
$$

where

$$
\begin{aligned}
& f_{1}=\left[(P-P a)+\rho v^{2} \frac{\sin (\phi-\theta) \cos \theta}{\sin \phi}\right] r \\
& f_{2}=\left[\rho v \frac{\sin (\varphi-\theta)}{\sin \phi}\right] r \\
& f_{3}=\cot \phi
\end{aligned}
$$

Maximizing I requires that the first variation of the integral must be equal to zero. In turn, this leads to an expression for the control surface and the flow conditions along it. The variaticn of I depends upon the optimization required. If the thinst is maximized such that the length is constant, but the area ratio varies, then the upper limit of the integral is a variable and contributes to the variation of $\mathrm{I}$. The variation of $I$ does not depend upon the lower limit.

Examining all possible variations of the quantities in I leads to the following set of pos sibilities:

(1) $\delta Z_{C} \delta M, \delta \theta$ and $\delta \phi$ are zero along $C D$ since this portion of the flowfield is fixed by the specification of the throat contour.

(2) $\delta M, \delta \theta$ and $\delta \phi$ are nonzero along $D E$.

(3) $\delta Z_{E}$ is zero at $E$, but $\delta r_{E}$ is nenzero.

(4) Since $M$ and $\theta$ are continuous along $C E$ then $\delta Z_{D}$ and $\delta r_{0}$, although nonzero, do not enter into the first variation of $\mathrm{I}$.

Performing the first variation of the integral $I$ results in

$$
\begin{aligned}
\delta I=0 & =\int_{r_{D}}^{r_{E}}\left[\left(f_{1}+\lambda_{2} f_{2}+\lambda_{3}^{1} f_{3}\right)^{\delta M}\right. \\
& +\left(1_{1} \lambda_{2}^{1}+\lambda_{3} f_{3}\right)^{\delta \theta} \\
& \left.+\left(f_{1}+\lambda_{2} f_{2}+\lambda_{3} f_{3}\right)^{\delta \phi}\right] \mathrm{dr} \\
& +\left.\left(1_{1}+\lambda_{2} f_{2}+\lambda_{3} f_{3}\right)\right|_{E} ^{\delta r_{E}}
\end{aligned}
$$

where the subscripts $M, \theta$ and denote partial differentiation and $\delta$ is the variational operator. Since the variations $\delta \mathrm{M}, \delta \theta, \delta \phi$ and $\delta r_{E}$ are arbitrary, each of their coefficients in Eq. (10) must be equal to zero. Thus,

$$
\begin{aligned}
& f_{1}+\lambda_{2} f_{2}+\lambda_{3} f_{3}=0 \\
& f_{1}+\lambda_{2} f_{2}+\lambda_{3} f_{3}=0 \\
& f_{1}+\lambda_{2} f_{2}+\lambda_{3} f_{3}=0
\end{aligned}
$$

along the line DE and

$$
f_{1}+\lambda_{2} f_{2}+\lambda_{3} f_{3}=0
$$

at $E$. If radial and axial distances at point $E$ are both specified, the upper limit in Eq. (10) is fixed and the last term in the equation disappears from the variational solution. Since $13_{M}=13_{\theta}=0$, these terms may be eliminated from Eqs. (11) and (12) and the two resulting equations combined to yield

$$
f_{1} f_{2}=f_{1} f_{\theta}^{2}
$$

The density, pressure and velocity can be considered functions of Mach number and computed from isentropic flow relations. Utilizing these relations it follows that

$$
\begin{aligned}
& \frac{d P / P_{T}}{d M}=P_{M}=-\frac{P^{2}}{M} \\
& \frac{d P}{d M}=P_{M}=-P M \\
& \frac{d V}{d M}=V_{M}=\frac{V}{M\left(1+\frac{r-1}{2} M^{2}\right)}
\end{aligned}
$$

The partial derivatives $f_{1}$ and $f_{2} M$ are determined from Eqs. (7) and (8) by making use of Eqs. (16)-(18). They are

$$
\begin{gathered}
f_{1}=\left[-\frac{P V^{2}}{M}+\frac{2 \rho v^{2} \sin (\varphi-\theta) \cos \theta}{M\left(1+\frac{\gamma-1}{2} M^{2}\right) \sin \phi}-\frac{V^{2} M P \sin (\varphi-\theta) \cos \theta}{\sin \varphi}\right] r \\
i_{2_{M}}=\left[\frac{\rho v}{M\left(1+\frac{\gamma-1}{2} M^{2}\right)}-V M P\right] \frac{\sin (\varphi-\theta)}{\sin \phi} r
\end{gathered}
$$


Similarly, the partial derivatives $f_{1}$ and $f_{2} \theta$ are

$$
\begin{aligned}
& f_{\theta}=-\frac{\rho v^{2}[\sin (\varphi-\theta) \sin \theta+\cos (\varphi-\theta) \cos \theta]}{\sin \varphi} r \\
& f_{2_{\theta}}=-\rho v \frac{\cos (\phi-\theta)}{\sin \phi} r
\end{aligned}
$$

Substituting Eqs. (19)-(22) into Eq. (15) and rearranging yields

$$
M^{2}=\frac{\cos (\phi-\theta)-\sin (\downarrow-\theta) \cos \theta \cos (\phi-\theta)+\sin ^{2}(\phi-\theta) \sin \theta}{\sin ^{2}(\phi-\theta) \sin \theta}
$$

By using simple trigonometric identities this may be reduced to

$$
M^{2}=\frac{1}{\sin ^{2}(\theta-\theta)}
$$

Since

$$
M=\frac{1}{\sin \mu}
$$

where $\mu$ is the Mach angle, it is apparent that

$$
\phi=\theta+\mu
$$

Therefore, the control surface is a left-running characteristic which implies that

$$
\frac{d r}{d z}=\tan (\theta+\mu)
$$

along DE. From Eq. (12)

$$
\lambda_{2}=-\frac{t_{\theta}}{i_{\theta}}
$$

Introducing Eq. (25) into Eqs. (21) and (22) and applying trigonometric identities gives

$$
\begin{aligned}
& t_{\theta}=-\rho v^{2} \frac{\cos (\theta-\mu)}{\sin \phi} r \\
& t_{2}=-\rho v \frac{\cos \mu}{\sin \phi} r
\end{aligned}
$$

Substituting these relations into Eq. (27) gives

$$
\lambda_{2}=-v \frac{\cos (\theta-\mu)}{\cos \mu}
$$

From Eq. (13)

$$
\lambda_{3}=-\frac{1_{1}^{+\lambda_{2} 1_{2}}}{1_{3}}
$$

The partial derivatives in this expression can readily be determined trom Eqs. (7) -(9) by employing Eqs. (16)(18). In turn, these may be substituted, along with Eq (30), 10 produce

$$
\lambda_{3}=\rho v^{2} \sin \theta\left[\frac{\cos \theta \cos \mu-\cos (\theta-\mu)}{\cos \mu}\right] r
$$

Using simple ingonometric identities in this expression brings

$$
\lambda_{3}=-\rho v^{2}\left(\sin ^{2} \theta \tan \mu\right) r
$$

Equations (25), (30) and (32) now yield the appropriate conditions to calculate the control surlace. The constants $\lambda_{2}$ and $\lambda_{3}$ can be evaluated from the properties at point D. According to Eq. (25), the control surface must coincide with a left-running characteristic. If this equation is satisfied then it follows that the compatibility equation for a left-running characteristic must also be satisfied along the control surtace line DE. The compatibility equation for axisymmetric isentropic irrotational flow, as given in Shapiro (4), is

$$
d \theta-\frac{\sqrt{M^{2}-1}}{M\left(1+\frac{\gamma-1}{2} M^{2}\right)} d M+\frac{\sin \mu \sin \theta d P}{\sin (\theta+\mu) r}=0
$$

It can be shown that this relation is satisfied by Eqs. (30) and (32).

In order for the nozzle to be optimum for a fixed length constraint, the condition in Eq. (14) must be satisfied at point E. Utilizing Eqs. (7) - (9), (30) and (32), Eq. (14) becomes

$$
\begin{gathered}
{\left[\left(P-P_{a}\right)+\rho v^{2} \frac{\sin \mu \cos \theta}{\sin (\theta+\mu)}\right]-\frac{v \cos (\theta+\mu)}{\cos \mu} \rho v \frac{\sin \mu}{\sin (\theta+\mu)}} \\
-\rho v^{2} \sin ^{2} \theta \tan \mu \cot (\theta+\mu) r=0
\end{gathered}
$$

This can be extensively reduced and shown to be the same as

$$
\frac{P+P a}{\frac{1}{2} P v^{2} \tan \mu}=\sin 2 \theta
$$

at point E. Equations (30) and (32) for $\lambda_{2}$ and $\lambda_{3}$ are 
used in conjunction with the craracteristic equations to define the flowfield and to relate the variables r. $Z, M$, and $\theta$ along a left-running (dasignated by the upper sign) or a right-running (designated by the lower sign) characteristic

$$
\begin{aligned}
& d \theta=\frac{\sqrt{M^{2}-1}}{{\sqrt{1+\frac{\gamma-1}{2} M^{2}}}^{2}} d M+\frac{1}{\left.\sqrt{\cot \theta} \sqrt{M^{2}-1-1}\right)^{d Z}} \\
& d r=\tan (\theta \pm \mu) d Z
\end{aligned}
$$

The equations are schematically shown in Fig. 6.

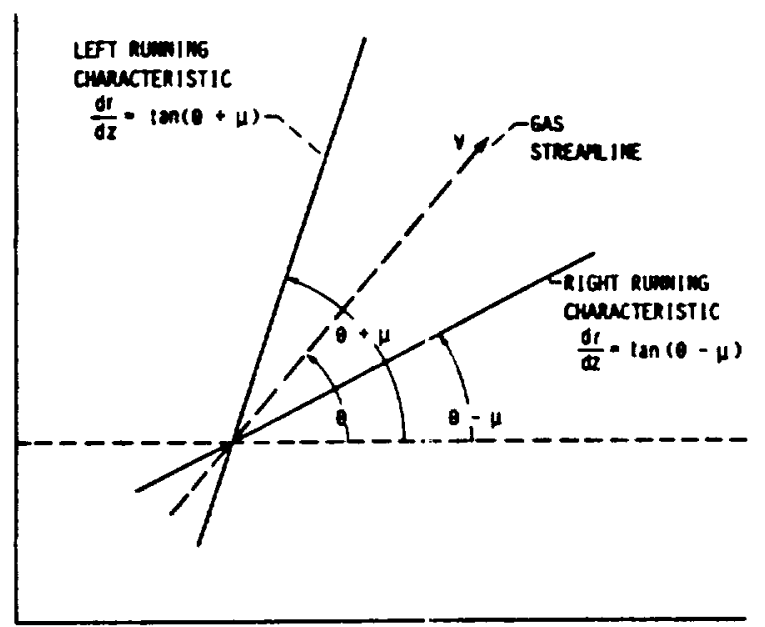

Flg. 6. - CHARACTERISTIC PLOT FOR TWO-DIMENSIONAL SUPERSONIC IRROT,ATIONAL FLOW

For two-dimensional Cartesiar geometries Eq. (32) is independent of $r$ and thus becones

$$
\lambda_{3}=-\rho v^{2} \sin ^{2} \theta \tan \mu
$$

Equations (25) and (30) remain the same as for axisymmetric flow and along with $\mathrm{Eq}$. (37) provide two equations in the two unknowns $M$ and $\theta$ and hence yield a constant $M$ and $\theta$ along the last characteristic. The character of the flow is therefore that of a simple wave region. This is in agreement with flow in the turning section of a two-dimensional Cartesian geometry nozzle.

\section{Scarfed Nozzle Truncation Poinl Computation}

In order to compute the contour of a truncaled scarted nozzle, the flow properties at "he cowl truncation point must be computed. A computer program was written to compute the Mach number, otlique shock wave angle, slip line angle Prandtl-Meyer expansion angles, along with the static and total pressures for this particular nozzle configuration. The computational procedure iterates on the flow angle and static pressure and repeats the computation until convergence is realized. The Mach number and static pressure behind the obllque shock wave and the oblique shock wave angle are computed and then used in the Rao program to construct the right-running characteristic at the cowl truncation point.

A schematic diagram of the nomenclature used in the scarted nozzle computer program for computing the flow properties at a truncation point on the nozzle cowl are shown in Fig. 7. Truncation of the nozzle to point F allows the extemal flow to affect the mechanics of the solution. Since the nozzle is assumed underexpanded, i.e., the internal static pressure is greater than the ambient static pressure, a Prandt-Meyer expansion fan and an oblique shock wave emanate from point $F$. Since the flow properties will differ in regions 2 and 2 ' in Fig. 7. a slip line also forms at point $F$ from the expansion of the internal flow. These flow phenomena are incorporated into the optimum nozzle program.

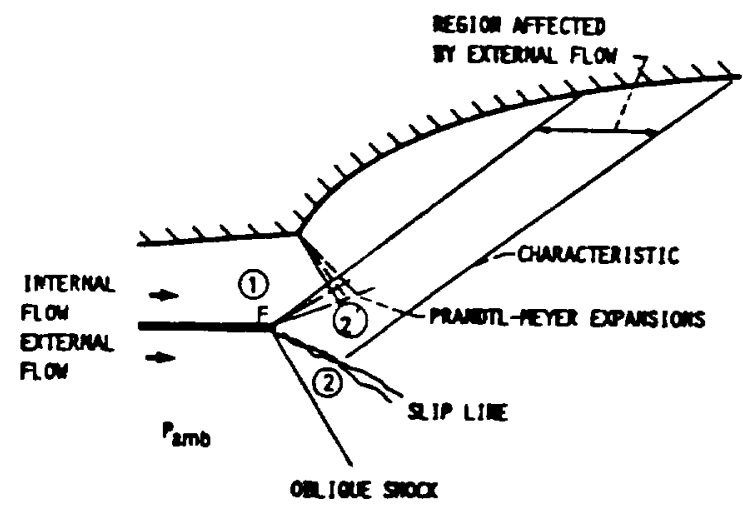

Fig. 7 - SCHEMATIC OF THE NOMENCLATURE USED IN SCARFED TRUNCATION POINT SUBROUTINE

Figure 8 is a flow diagram of the scarted nozzle program. This computer code determines the phenomena which occur at the cowl truncation point. Data is input for the ambient and nozzle Mach numbers $M_{a}$ and $M_{1}$ respectively, specific heat ratios and static pressures. A check is performed on the ambient Mach number to determine whether its value is greater than 1.0 If $\mathrm{M}_{\mathrm{a}}$ is supersonic, the static pressure behind the Prandtl-Meyer expansion is initialized by setting it equal to the ambient static pressure. If on the other hand $M_{2}$ is subsonic, the static pressure behind the Prandtl-Meyer expansion is initialized by computing the average of the ambient and nozzle static pressures. The flow regions computed by the scarted nozzle program are numbered as shown in Fig. 7 .

Total pressures for the ambient flow and flow regions 1 and 2 along with the Prandt-Meyer expansion angle $v$ at state point 1 are computed from isentropic flow equations. The total pressure within state 2 is set equal to the total pressure at state 2' because the total pressure across the slip line is constant. The Mach number at state 2 is computed next from the total to static 


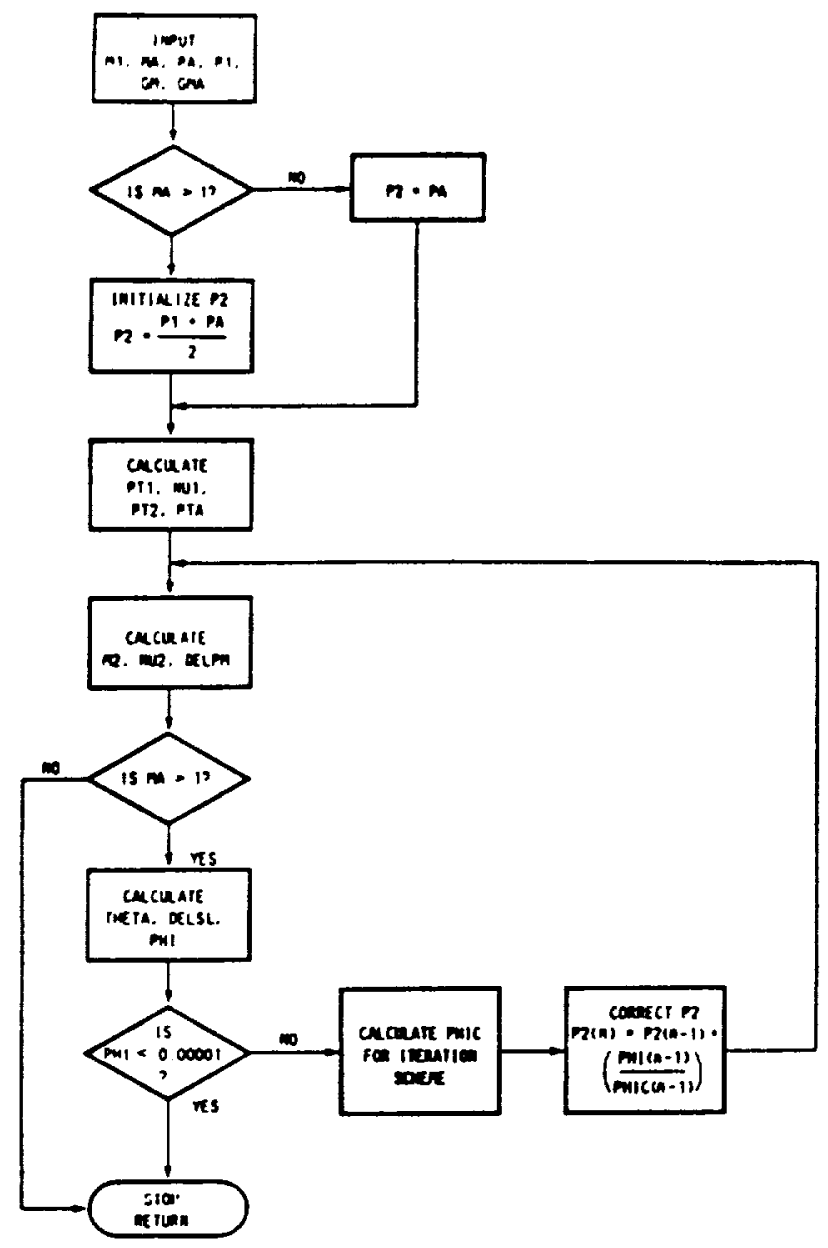

Fig. 8 - COMPUTER FLOW DIAGRAM FOR THE SCARFED TRUNCATION POINT SUBROUTINE

$$
\left.\cot \delta=-\tan \theta \frac{(\gamma-1) M_{1}^{2}}{2\left(M_{1}^{2} \sin ^{2} \theta-1\right)}-1\right]
$$

The oblique shock angle is obtained from

$$
\frac{P_{2}}{P_{1}}=\frac{2 \gamma}{\gamma+1} M_{1}^{2} \sin ^{2} \theta-\frac{\gamma-1}{\gamma+1}
$$

pressure ratio along with the corresponding Prandt1Meyer expansion angle. The total Prandtl-Meyer expansion angle is then computed by determining the absolute value of the difference in expansion angles at states 1 and 2 .

The next step in the scarfed nozzle subprogram is to perform a test on the ambient Mach number to establish its value. For ambient Mach numbers less than 1.0, the subroutine returns to the main Rao program. For ambient Mach numbers greater than 1.0. the slip line angle and the oblique shock angle are computed. The slip line angle is computed from
The absolute value of the difference of the total Prandtl-, Meyer expansion angle and the oblique shock angle are computed and checked for convergence. A difference of 0.00001 between successive passes was used as a convergence criteria. If the convergence test tails, the subroutine computes derivatives of Eqs. (38) and (39) as well as those for $v$ and $P_{T} / P$. These are then used in Newton's method for iterative solution of simultaneous equations as described in Press et al (5)

The oblique shock angle, state 2 Mach number and the static pressure are next us'ed in the Rao program for constructing the right-running characteristic at the truncation point.

\section{Slip Line and Oblique Shock Wave Computation}

The oblique shock wave and slip line that occur at the cowl truncation point may be curved or straight depending upon the magnitude of the static pressure difference between the ambient and the nozzle flows. In general, the oblique shock wave and the slip line will be curved because of the additional expansion which takes place in the internal nozzle flow. If the oblique shock wave is curved, the strength of the shock will vary from point to point. The flow downstream of the shock is only isentropic along streamlines and is rotational because of gradients in entropy and stagnation properties normal to the streamlines. For isentropic flows the entropy is by definition constant along streamlines.

Zucrow and Holfman (6) develop the equations which govem steady adiabatic flow of an inviscid compressible fluid which are used to derive the characteristic equations for a rotational flow. Figure 9 shows a plot of the characteristics for steady two-dimensional supersonic rotational flow. The characteristic directions and compatibility equations derived for the Mach lines

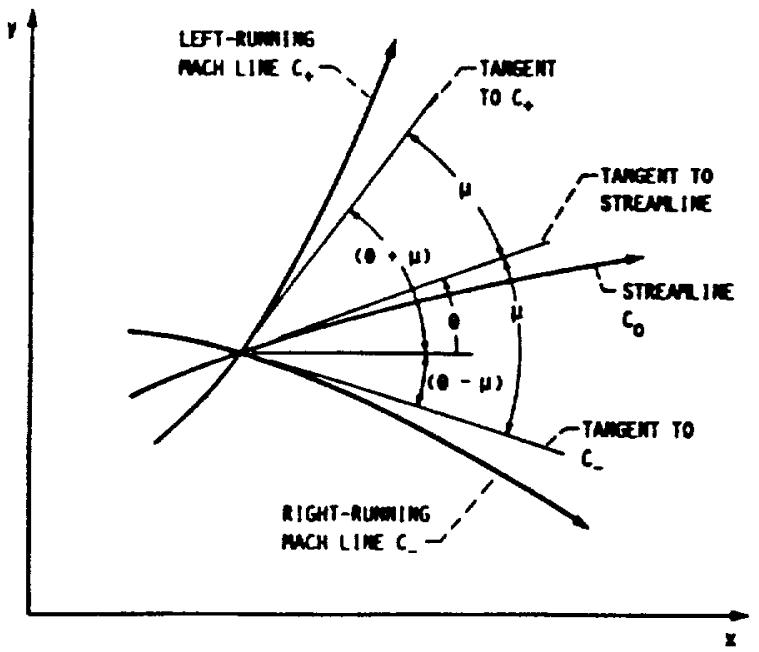

Fig. 9 - CHARACTEAISTIC PLOT FOR STEADY TWO DIMENSIONAL SUPERSONIC ROTATIONAL FLOW 
remain the same as those for imstational flow. However, two additional equations are obtained. These are the streamline (counted iwice) and the compatibility relations along the streamlines. The compatibility equations are the Bernoulli equation and the speed of sound relation.

The finite difference equations for the rotational flow relations are developed in Ref. (7) along with equations for interior point and external point shock wave calculation.

\section{RESULTS AND DISCUSSION}

The flow within a inuncated scart nozzle was calculated for a design Mach number of 6, i.e., the Mach number at the last nozzle characteristic, with an external flow Mach number of 5 . The specific heat ratio for both internal and external flows was taken to be 1.4. An area ratio of 90. nozzle ramp length of 300 inches, nozzle cowl length of 15 inches, nozzle throat Mach number of 1.24, nozzle throat temperature of $2500^{\circ} \mathrm{R}$, along with ambient static pressure of 0.150 psia and 100 ssia for the nozzle throat total pressure completes the input used for this design. Pressures and coordinates computed in this analysis are nondimensionalized with respect to the nozzle throal conditions.

A nontruncated scarted nozzly was also designed to compare the effect of truncat on. The nontruncated nozzle was computed for the same design Mach number as the iruncated nozzle (the extisnal flow does not affect the nozzle computation, as shown in Fig. 2). The nontruncated nozzle computation requires approximately 10 seconds pf CPU time on an IBM 3033 mainframe computer, while the truncated scarted case requires approximately twice the amount of CPU time.

Figure 10 is a plot of non-dimensional length (ratio of nozzle length to nozzle throat height) versus nozzle thrust coefficient computed rom the modified Rao computer program. The cowl for the scarted nozzle, designed for a Mach number of 6. was selectively truncated for lengths ranging from 50 to 12.5. A nozzle

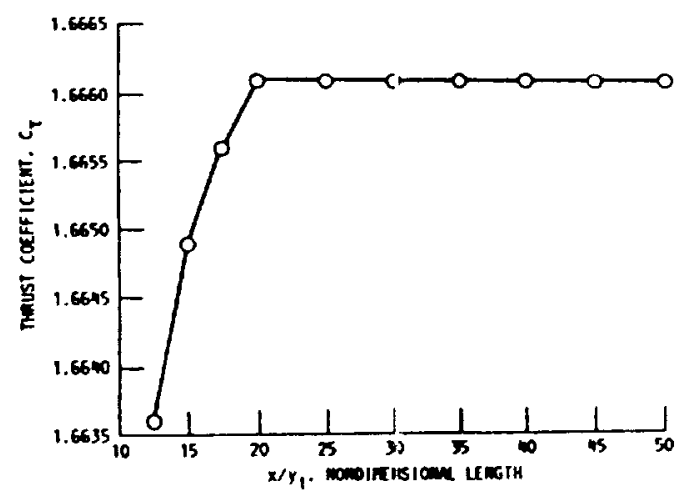

Fig. 10 - PLOT OF NOZZLECOW LENGTH VERSUS NOZZLE THRUST COEFFICIENT vacuum thrust coefficient of 1.666 was computed for the non-truncated case and is invariant with cowl truncation to a non-dimensional length of 20 . Truncating the cowl beyond this value causes the thrust coefficient to drop. sharply as shown in Fig. 10. A nozzle cowl length of 20 corresponds to the condition in which the last nozzle ramp characteristic intersects the cowl surface as illustrated in Fig. 2. Figure 10 clearly displays the moditication made to the downstream boundary condition for the iruncation point in the Rao program.

The nontruncated nozzle and the truncated scarfed nozzle were then analyzed with the Seagull computer code, developed by Salas (8). These computations were performed on the Cray XMP supercomputer and required approximately 30 seconds of CPU time per case.

Figures 11 to 15 are respectively plots of flow path geometry, static pressure, axial velocity, axial Mach number and Mach number contours for the nontruncated nozzle. Figures 12 and 13 display smooth distributions of static pressure and axial velocity. The Mach number

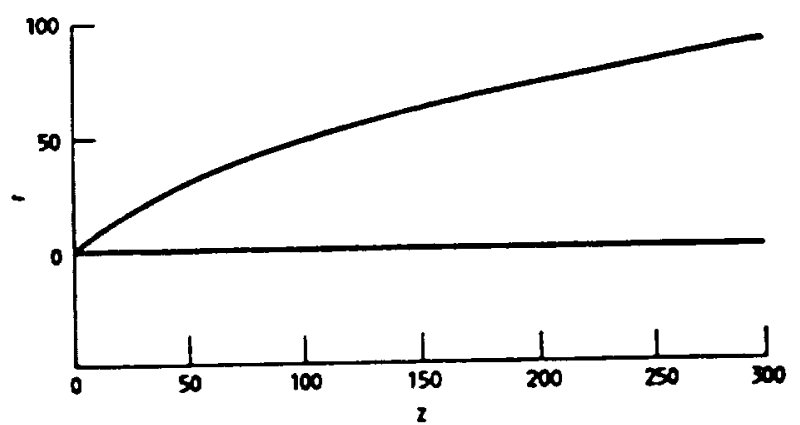

Fig. 11 - FLOW PATH GEOMETRY PLOT OF NONTRUNCATED NOZZLE

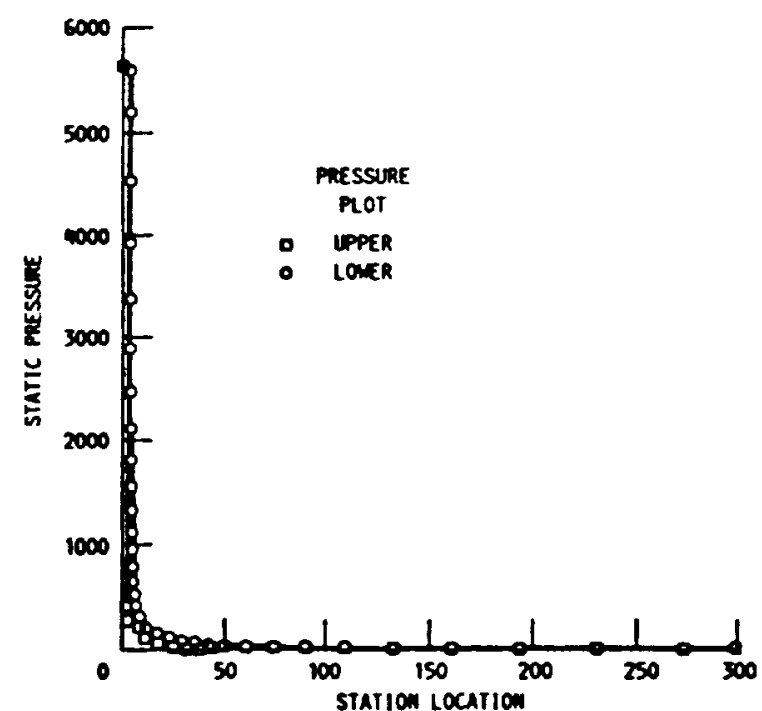

Fig. 12 - WAL STATIC PRESSURE PLOT OF NONTRUNCATED NOZZLE 


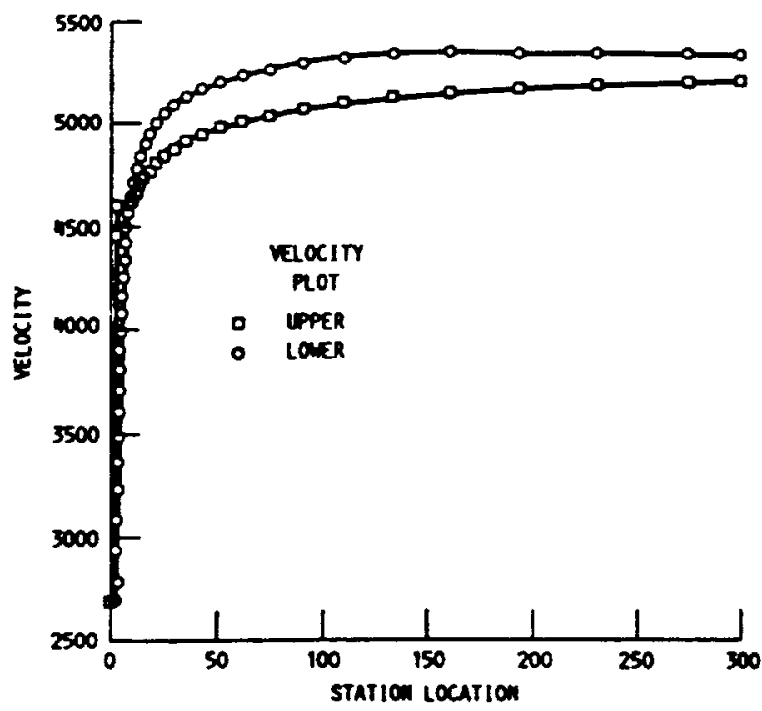

Fio. 13 - WALL AXIAL VELOCTY PLOT OF NONTAUNCATED NOZZLE

distribution in Fig. 14 shows a wave in the lower wall Mach number. This wave can be attributed to the difference in the upstream boundary conditions assumed at the throat in the Rao and Seagull programs. The Rao program assumes a curved sonic line at the throat, whereas the Seagull program assumes the throat sonic line is straight. The Seagull program computes this wave as a Prandtl-Meyer expansion fan. This is clearly depicted in the Mach number contour plot in Fig. 15.

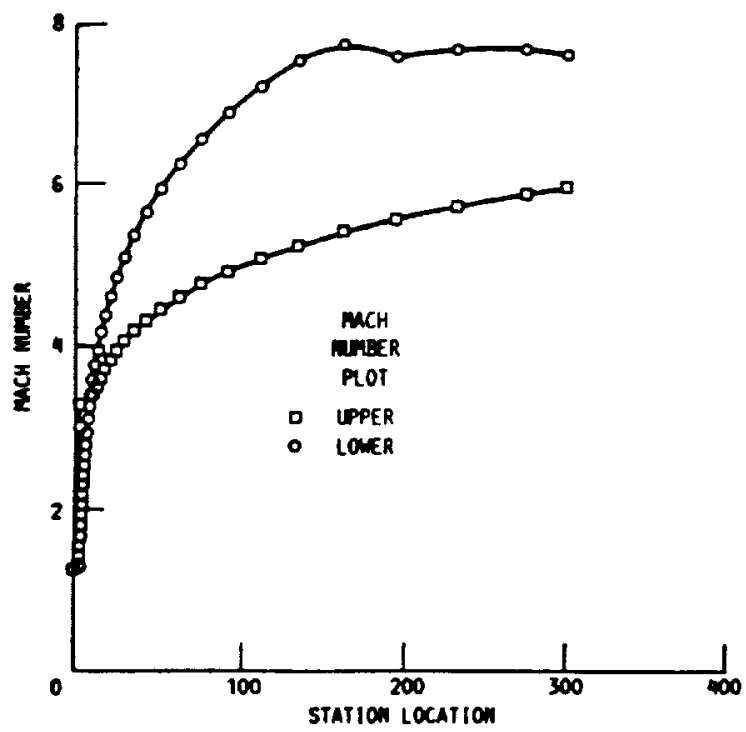

Flo. 14 - WALL AXIAL MACH NUMBER PLOT OF NONTRUNCATED NOZZLE

The analysis of the inuncated scarfed nozzle case are presented in Figs. 16 to 21. Figure 16 is a plot of the flow path geometry with the cowl truncated at a nondimensional length of 15. The solid line extending past this point is a boundary drawn by the Seagull program. Figure 17 contains the static pressure plot of the inuncated scarfed nozzle and shows an abrupt drop in the static pressure distribution on the lower wall at the

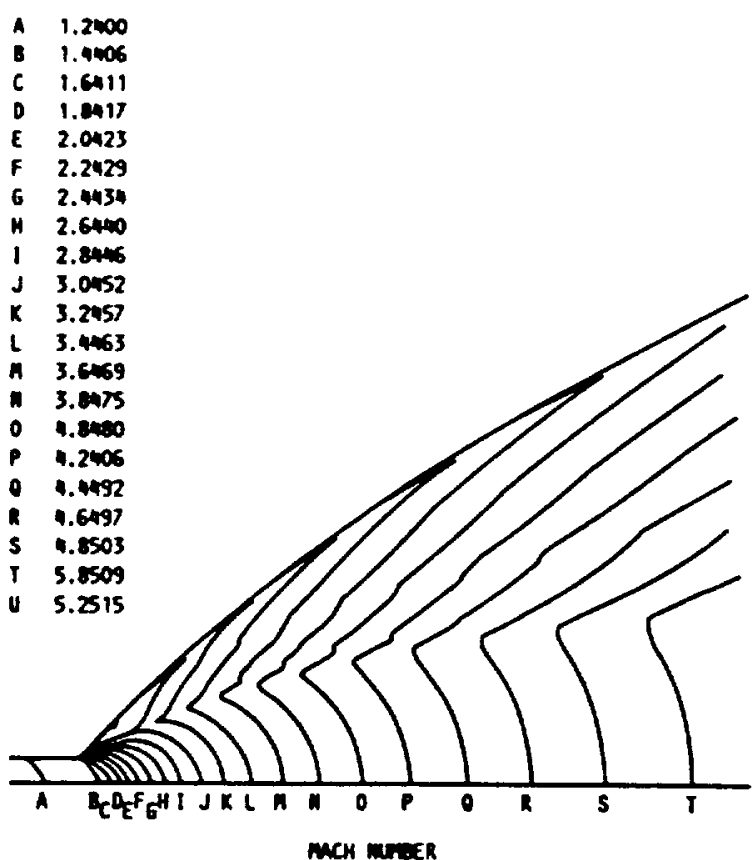

Fig. 15 - MACH NUMBER CONTOUR PLOT OF NONTRUNCATED NOZZLE

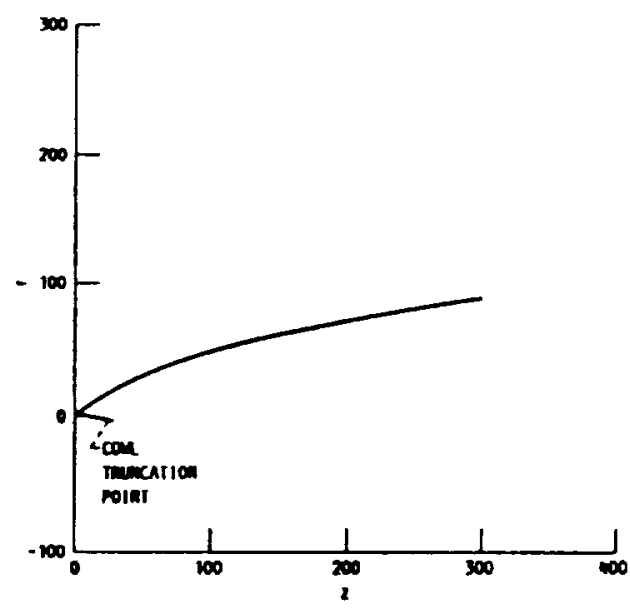

Fig. 16 - FLOW PATH GEOMETAY OF TRUNCATED SCARFED NOZZLE

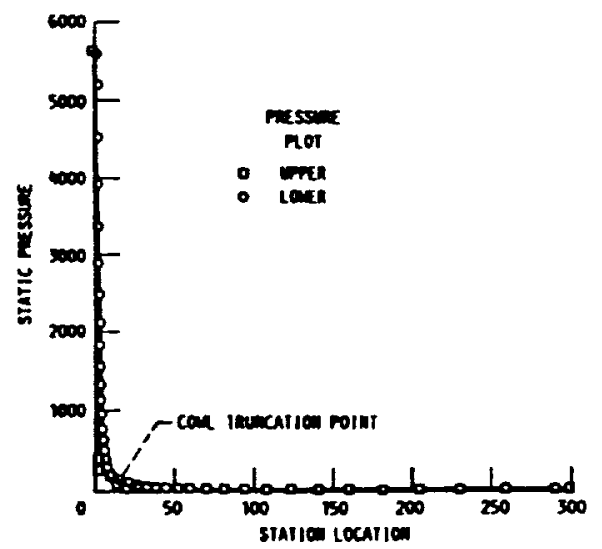

Fig. 17 - WALL STATIC PRESSURE PLOT OF TRUNCATED SCARFED NOZZLE 
truncation point. The lower wall static pressure becomes equal to the freestrear static pressure slightly beyond the truncation point.

Figure 18 displays the computed axial velocity distribution. As exhibited ir the static pressure distribution, the axial velocity drops to the freestream velocity beyond the truncation point. The Mach number distribution also displays a similar behavior and shows that the tuncated scarled nozzle does not internally expand as much as the nontruncated nozzle. This clearly illustrates the effect of the external flow on the expansion of the internal flow as shown in Fig. 19.

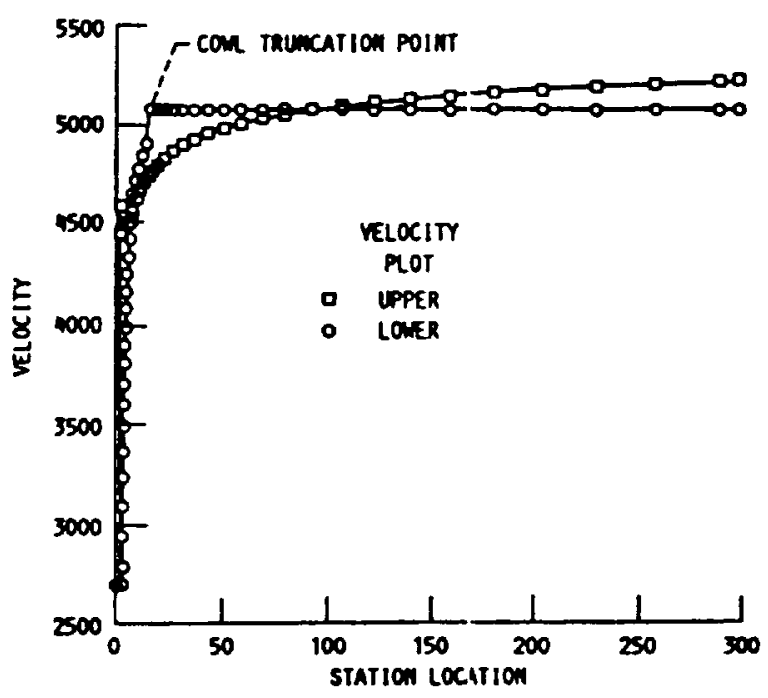

Fig. 18 - WALL AXIAL VELOCITY PLOT OF TRUNCATED SCARFED NOZZLE

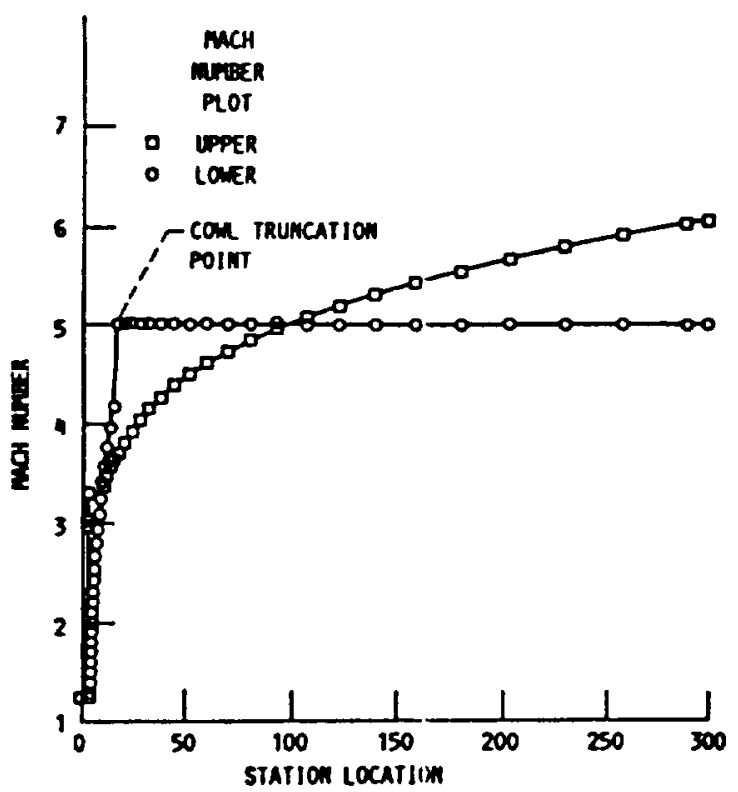

Fig. 19 - WALL AXIAL MACH NUMBIER PLOT OF TRUNCATED SCARFED NOZZLE
Figures 20 and 21 are plots of the oblique shock wave and the slip line and Mach number contours for the inuncated scarted nozzle. Figure 20 displays the oblique shock wave and slip line emanating from the cowl truncation point. The slip line initially diverges from the internal flow and then converges as the effect of the external flow increases with nozzle length. The Mach number contours plotted in Fig. 21 clearty illustrates the interaction of the external and internal flows. The slip ine is depicted by the coalescing of contour lines in the center of the plot and the strength of the oblique shock wave is shown in the bottom portion of the figure. Also. the Prandtl-Meyer expansion fan interacts with the slip lne in this case instead of the nozzle cowl wall as in the nontruncated case.

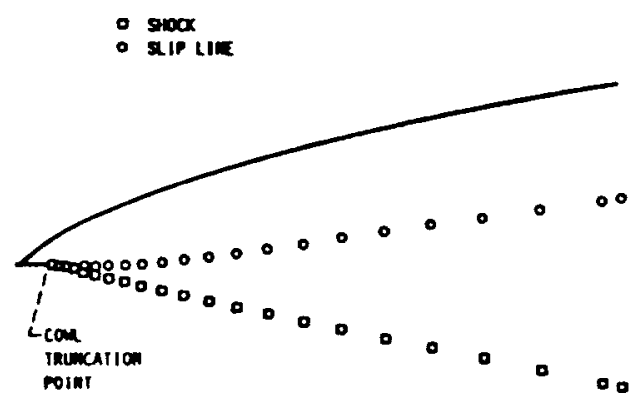

Fig. 20 - OBLIQUE SHOCK WAVE AND SHEAR LAYER PLOT OF TRUNCATED SCARFED NOZZLE

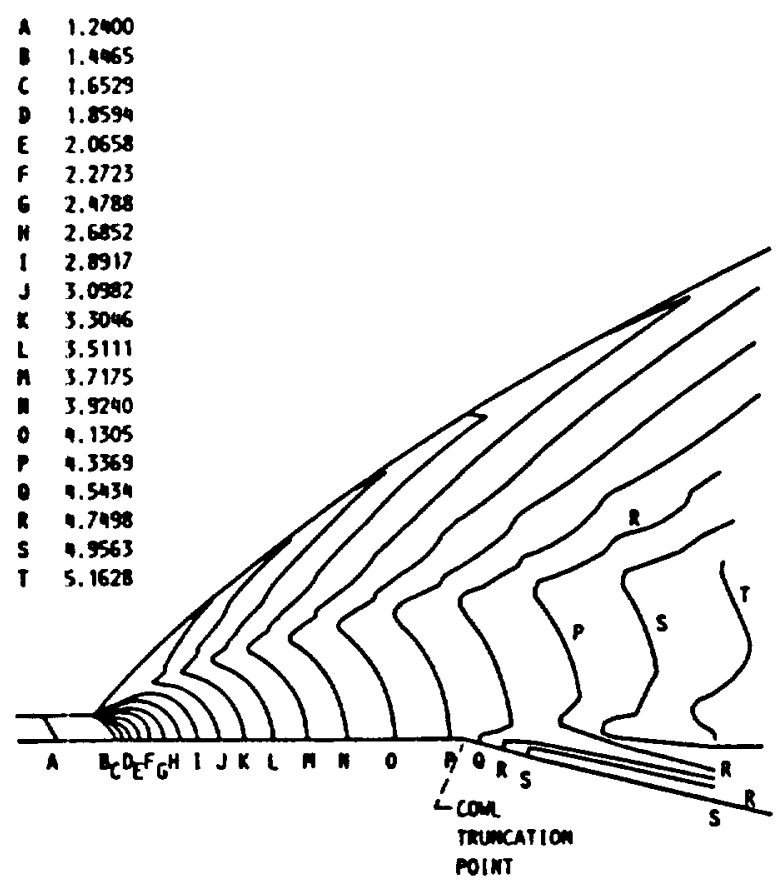

Mach munen

FI. 21 - MACH NUMBER CONTOUN PLOT OF TRUNCATED SCARFED NOZZLE

\section{ORIGIRAL PAGE IS \\ OF POOR QUALITY}


Figure 22 is a comparison of nozzle ramp wall Mach number distributions for the truncated scarfed nozzle. The Rao design and the Seagull analysis compare very favorably except for the corner region where the compression wave occurs. This is illustrated by the jump in Mach number at approximately 2 inches as shown in Fig. 22.

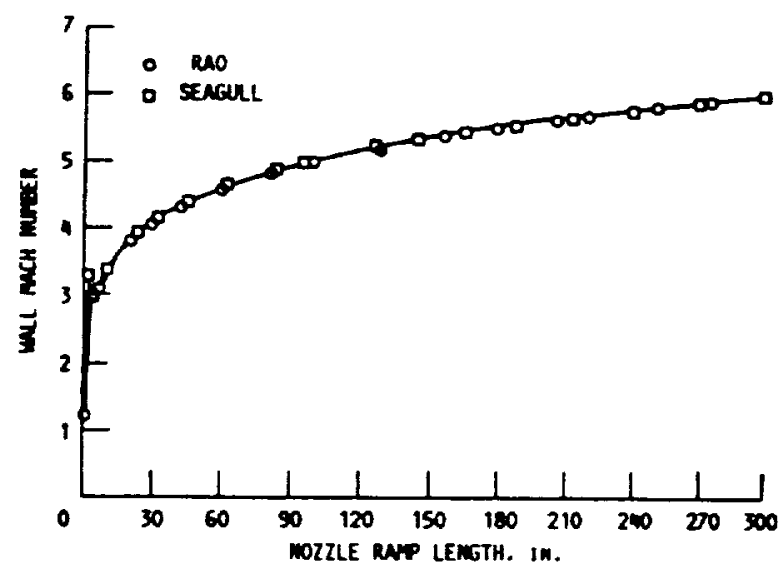

FIG 22 - COMPARISON OF NOZZ E RAMP WALL MACH NUMBER DISTRIBUTIONS

\section{CONCLUDING REMARKS}

Results of a calculation of an optimized truncated scarled nozzle were compared with values for a nontruncated version. The truncated scarfed nozzle design showed less expansion than did the nontruncated case and this was primarily due to the external flow affecting the internal flow expansion within the nozzle.

The comparison of thrust coefficient versus nozzle cowl length revealed that truncation of the cowl will affect the overall performance of an exhaust nozzle. This comparison demonstrates that there is an optimal cowl length in which truncation can be performed without degrading the overall nozzle pertormance. Truncation of the cowl past this optimal length should be analyzed in trade-off studies for thrust loss versus gross vehicle weight.

The truncated scarfed nozzle method developed in this paper can be extended in the future. The method can be moditied to compute an optimum truncated scarted nozzle with an oblique shock wave forming internally and simple chemical kinetics could also be incorporated into this method.

\section{REFERENCES}

1. Rao, G.V.R., Exhaust Nozzle Contour for Optimum Thrust," Jet Propulsion, Vol. 28, No. 6, June 1958, pp. 377-382.
2. Nickerson, G.R.,"The Rao Optimum Nozzle Program," SEA Report No. 6/82/800.1. Software \& Engineering Association, Santa Ana, CA, June 1982.

3. Hildebrand, F.B., Methods of Applied Mathematics, 2nd Edition, Prentice-Hall, 1965.

4. Shapiro, A.H., The Dynamics and Thermodynamics of Compressible Fluid Flow, Vol. I, Ronald Press, New York, 1953.

5. Press, W.H., Flannery, B.P., Teukolsky, S.A. and Vetterling, W.T., Numerical Recipes, Cambridge University Press, New York, 1986.

6. Zucrow, M.J. and Hoftman, J.D., Gas Dynamics: Muttidimensional Flow, Vol. II, Robert Krieger Publishing Company, Malabar, FL, 1977.

7. Shyne, R.J., "Analysis and Optimization of Scart Nozzles Subject to External Flow Conditions," NASA TM-100955, August 1988.

8. Salas, M.D., "Shock Fitting Method for Complicated Two-Dimensional Supersonic Flows," AIAA J. Vol. 14, No. 5, May 1976, pp. 583-588. 


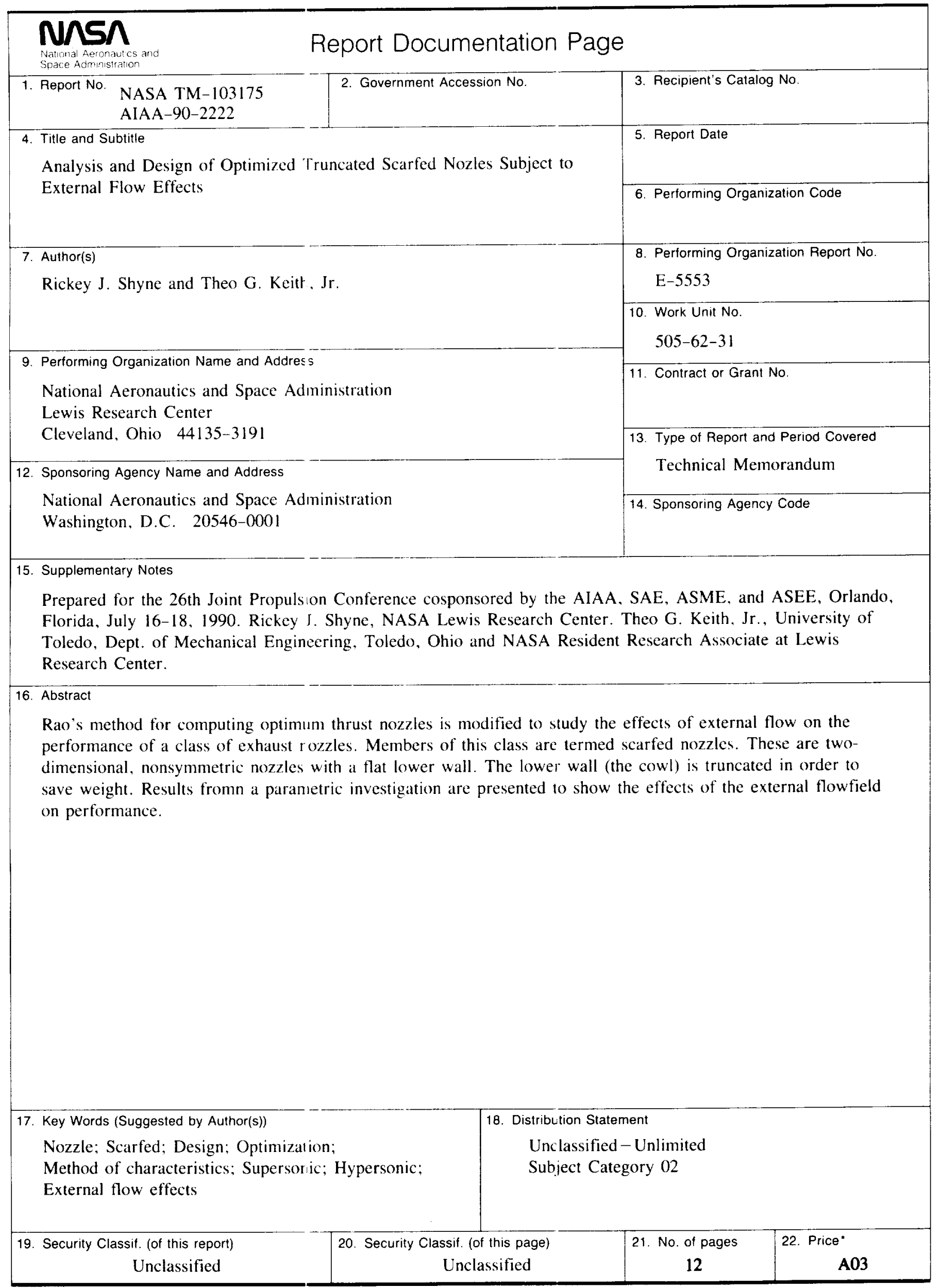


